\section{Molecules in Glass: Probes, Ordered Assemblies, and Functional Materials}

BRUCE DUNN ${ }^{*, \dagger}$ AND JEFFREY I. ZINK ${ }^{*,+}$

Departments of Materials Science and Engineering and Chemistry and Biochemistry, University of CaliforniaLos Angeles, Los Angeles, California 90095

Received February 14, 2007

\section{ABSTRACT}

Research on the properties and applications of molecules doped into sol-gel-derived silica matrices has expanded rapidly. This Account begins with a brief review of the use of the dopant molecules as probes of the changes that occur as the system evolves from the initial sol to the final xerogel during the formation of monoliths, thin films, and mesostructured films. Methods of deliberately placing desired molecules in specific regions of the mesostructure are discussed, and an application, energy transfer, is presented. Finally, encapsulation of biological molecules is examined, and two important aspects, stabilization of the biomolecules and applications as biosensors, are described.

\section{Introduction}

The sol-gel process is a simple, bench-top chemical synthesis method for forming inorganic glass at room temperature. The use of alkoxysilane precursors to prepare transparent silicate glass can be traced back to the 19th century. Because of the low temperature and mild synthetic conditions, molecular dopants that are impossible to add to traditional glasses because of the high melting temperatures can be incorporated in sol-gel-derived materials. Although studies as early as 1949 demonstrated that organic dyes could be incorporated in a silica matrix prepared at room temperature, ${ }^{1}$ it was not until the $1980 \mathrm{~s}$ that systematic studies based on alkoxysilanes began. ${ }^{2}$ As interest expanded, the research evolved into two complementary directions: (1) the incorporation of organic

Professor Bruce Dunn holds the Nippon Sheet Glass Chair in Materials Science and Engineering at the University of California - Los Angeles (UCLA). He received his B.S. degree at Rutgers University and his M.S. and Ph.D. degrees at UCLA. He was a staff scientist at the General Electric Corporate Research and Development Center before joining the UCLA faculty in 1980. His research interests concern the synthesis of ceramics and inorganic materials and characterization of their electrical, electrochemical, and optical properties. A continuing theme in his research is the use of sol-gel methods to synthesize materials that incorporate specific dopants and are capable of developing unique microstructures and properties. The areas presently being studied in his group include biosensors, intercalation compounds, aerogels, and organic/inorganic hybrid materials.

Jeffrey I. Zink is a Distinguished Professor of Chemistry in the Department of Chemistry and Biochemistry and a member of the California Nanosystems Institute at UCLA. He received his B.S. degree at the University of Wisconsin and his $\mathrm{Ph}$.D. degree at the University of Illinois Urbana-Champaign under the supervision of Russell S. Drago. After graduation, he joined the faculty at UCLA as an Assistant Professor. His research interests include excited-state properties of metalcontaining molecules (especially excited-state distortions and excited-state mixed valence) studied by electronic and resonance Raman spectroscopy and the timedependent theory of spectroscopy, triboluminescence, sol-gel optical and biomaterials, and nanostructured functional materials. molecules as spectroscopic probes of sol-gel chemistry and structure and (2) the development of materials with functional optical properties. The overwhelming success of this work led researchers to explore other systems, in particular, biomolecules.

In this Account, we review the evolution of a field based on the incorporation of dopant molecules in sol-gel-derived matrices. We begin with the work on spectroscopic probes of the physical and chemical changes that occur during sol-gel processing. We continue with studies of thin films, meso-structured materials with ordered arrays of templated pores, and the deliberate placement of active molecules within mesostructured materials. The development of materials that exhibit designed optical properties, on the basis of the added dopant, is discussed briefly because other contributors to this special issue cover that topic in far greater detail. We conclude with a review of biomolecule encapsulation in sol-gel matrices.

Preparation of Inorganic Glasses by the Sol-Gel Method. The synthesis of materials by the sol-gel process generally involves the use of metal alkoxides, which undergo hydrolysis and condensation polymerization., One of the advantages of the sol-gel process is the versatility of fabricating the materials derived from this process into different forms, including bulk monoliths, fibers, and films. The overall reactions leading to the formation of silica are

$$
\begin{gathered}
\mathrm{Si}(\mathrm{OR})_{4}+4 \mathrm{H}_{2} \mathrm{O} \rightarrow \mathrm{Si}(\mathrm{OH})_{4}+4 \mathrm{ROH} \\
n \mathrm{Si}(\mathrm{OH})_{4} \rightarrow n \mathrm{SiO}_{2}+2 n \mathrm{H}_{2} \mathrm{O}
\end{gathered}
$$

The sol-gel process can ordinarily be divided into the following steps: forming a solution, gelation, aging, drying, and densification. In the preparation of a silica glass, an appropriate alkoxide [e.g., tetraethoxysilane (TEOS), $\mathrm{Si}\left(\mathrm{OC}_{2} \mathrm{H}_{5}\right)_{4}$, or tetramethoxysilicate (TMOS), $\left.\mathrm{Si}\left(\mathrm{OCH}_{3}\right)_{4}\right]$ is mixed with water and a mutual solvent, such as ethanol or methanol, to form a solution. Hydrolysis leads to the formation of silanol groups, $\mathrm{Si}-\mathrm{OH}$. These species are only intermediates because they react further to form $\mathrm{Si}-\mathrm{O}-\mathrm{Si}$ groups. As the hydrolysis and condensation polymerization reactions continue, viscosity increases until the solution ceases to flow. This sol-gel transition is irreversible, and at this stage, the one-phase liquid is transformed to a two-phase system. The gel consists of amorphous primary particles of variable size $(5-10 \mathrm{~nm}$ or smaller) with an interstitial liquid phase. At this stage, the pores have yet to shrink and the liquid phase fills the pores. After gelation, gels are generally subjected to an aging treatment. Condensation reactions continue, increasing the degree of cross-linking in the network. The aging process is followed by drying, which involves the removal of the liquid phase and the formation of a xerogel. Ambienttemperature evaporation is frequently employed, and there is considerable weight loss and shrinkage. It is at

* To whom correspondence should be addressed. E-mail: zink@chem.ucla.edu.

† Department of Materials Science and Engineering.

‡ Department of Chemistry and Biochemistry. 


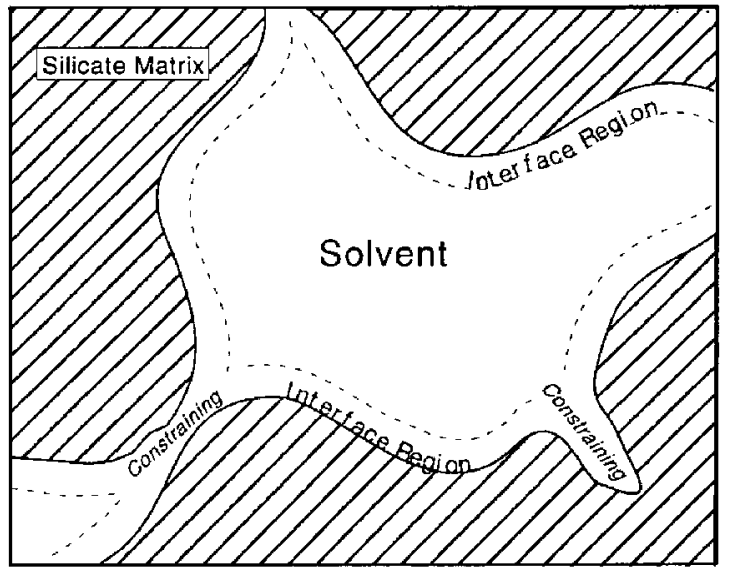

FIGURE 1. Representation of the four locations for dopant molecules in sol-gel materials: the interior of the pore, the interface between the solvent and the pore wall, the pore wall, and the constraining region. ${ }^{5}$

this stage that pore collapse occurs, decreasing the pore size and thus decreasing the solvent volume.

\section{Spectroscopic Probes of the Sol-Gel Process}

Spectroscopic probes have been used to characterize the chemical and physical changes that occur during the various stages of the sol-gel process. ${ }^{5}$ In the use of spectroscopic probes of the sol-gel process, a critical question to be considered is the location of the probe molecule because this determines the environment to which it is exposed. Four general locations of the sol-gel matrix are identified in Figure 1. As reviewed below, the various probe studies provide insight concerning the identity or composition of the solvent, its polarity, viscosity, the $\mathrm{pH}$ of the solvent (if it includes water), and the rates of chemical reactions. The influence of the pore walls may also be very important, even when the molecule is dissolved in a solvent contained in the pore.

Pyranine (8-hydroxy-1,3,6-trisulfonated pyrene) has emerged as an extremely useful spectroscopic probe for sol-gel processes. ${ }^{6,7}$ Pyranine was first used as a probe to detect solvent chemistry changes in TMOS and aluminosilicate sol-gels during sol formation and gelation. The luminescence peaks for the protonated and deprotonated forms are different, enabling a luminescence ratio method to be used to determine the water/alcohol ratio in the sol and the pore solvent (Figure 2). These experiments demonstrated that changes in gel chemistry occur well beyond the gelation stage. Another important result was the discovery that the trapped pyranine communicated through pores with the environment outside of the glass, which has led to the use of doped sol-gels as chemical sensors.

A different spectroscopic probing method, fluorescence polarization, has been useful in detecting the onset of gelation. ${ }^{8,9}$ Fluorescence polarization measures the rotation of a molecule in a sol-gel matrix by monitoring the reorientational movements of molecules. One of the most important results of these studies was the interpretation<smiles></smiles>

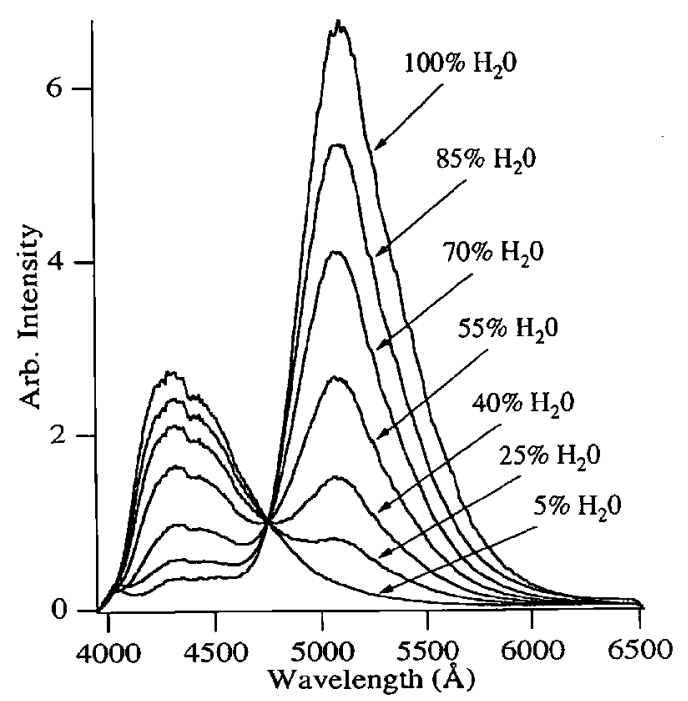

FIGURE 2. Pyranine has been widely used as a probe molecule to detect solvent changes during sol-gel processing. The structure of the protonated form of pyranine (the dominant form in alcohol) is shown on the top. On the bottom is shown how the changes in the luminescence spectra of pyranine vary as a function of the ethanol/ water ratio. ${ }^{7}$

of sol-gel "domains" of "constant", "low", "intermediate", and "high" microviscosities. The "constant" domain corresponds to the pore solvent region in which the molecule has a reorientation time similar to that in a solution in a beaker. The other microviscosity domains probably correspond to the interface, pore wall, and constraining regions.

Rigidochromism provides a probe method that measures the reorientation of solvent dipoles around the probe. The luminescence rigidochromism of (2,2-bipyridine)tris(carbonyl)-chlororhenium(I), $\mathrm{ClRe}(\mathrm{CO})_{3}$-2,2-bipyridine, was used to probe aging and drying processes at the nanodimensional level, which occur during the sol-gel-xerogel processes of TEOS silicates and aluminosilicates. ${ }^{10}$ In the silicate sol-gel system formed from TEOS, the solvent molecules remain unconstrained in an interstitial liquid phase until the final stages of drying.

\section{Spectroscopic Probes of Thin-Film Formation and Dynamics}

Silica Films. Sol-gel thin films are technologically important because of their applications in optical and electronic coatings. ${ }^{3,4}$ We successfully applied our spectroscopic probe methods to sol-gel-film formation. ${ }^{11}$ In 


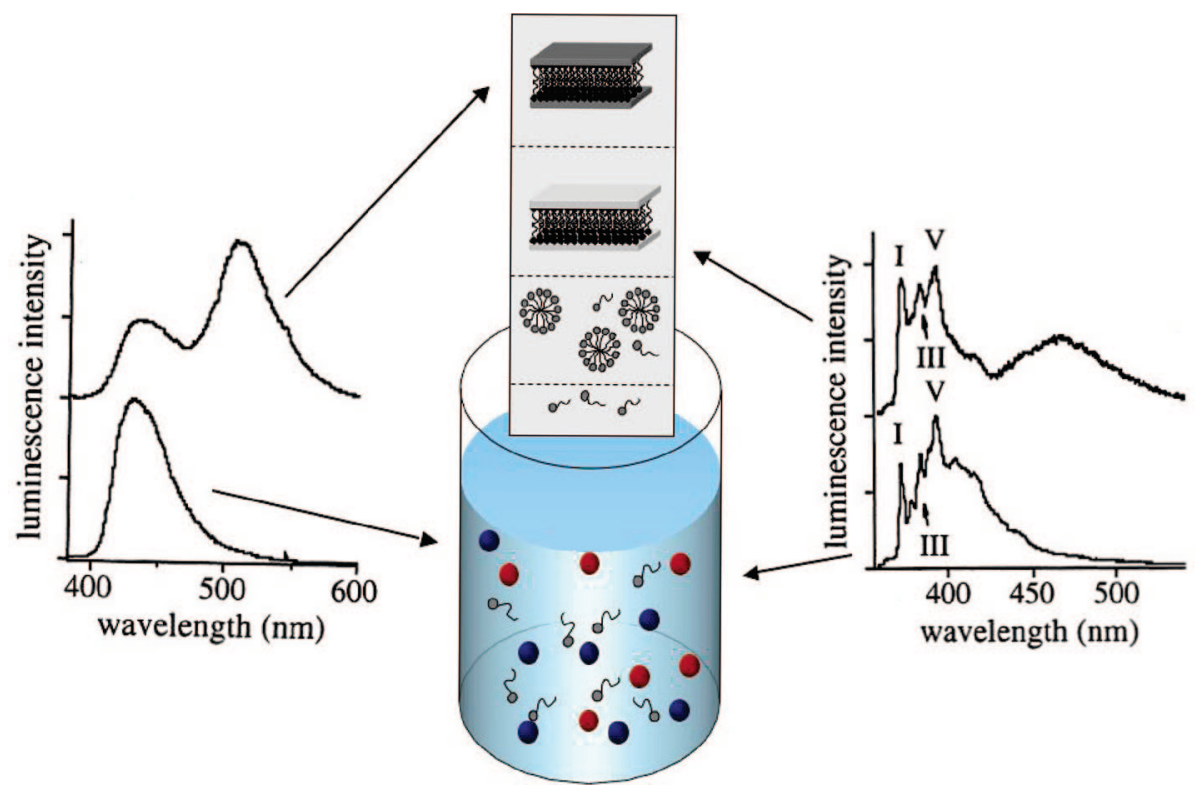

FIGURE 3. Pictorial representation of the mesostructure-film formation process. The spatially and temporally separated steps are micelle formation, network formation, and silicate condensation that keeps the mesostructure intact. The spectral changes of pyranine (left) monitor the preferential evaporation of alcohol during deposition. The spectral changes of pyrene (right) monitor micelle formation.

this work, we were able to characterize the chemical and structural evolution using real-time, in situ monitoring of spectroscopic probe molecules.

We focused on films formed by the dip-coating process, in which a substrate is withdrawn slowly at a constant speed from a sol that contains mono- or oligomeric silicate species. ${ }^{11}$ The moving substrate entrains the sol forming the liquid film. This film thins by solvent evaporation, gravitational draining, and possibly surface-tension-gradient driven flow. In our first studies, we combined interferometry with luminescence spectroscopy of pyranine to establish that preferential evaporation of alcohol occurs during film deposition, leading to the formation of a water-rich film that subsequently dries to form the deposited film. The structural evolution during film deposition is very complex and rapid; the sol-gel-xerogel transformation is complete within $30 \mathrm{~s}$. The result is that the drying stage overlaps with hydrolysis and condensation reactions. The use of interferometry is an important part of this experiment because it allows us to measure the changing thickness of the film as it is being formed on the substrate. When both interferometry and fluorescence depolarization are used simultaneously, the results show that probes tumble freely throughout much of the dip-coating process, suggesting that oligomer aggregation forms an extensive silicate framework. In aged sols, however, probe motion is constrained to a much greater extent because there is a higher degree of condensation/ oligomer formation in the sol.

Mesostructured Films. Recent developments in the preparation of surfactant-templated mesostructured sol-gel silica materials have extended the morphology from the originally discovered powders, with particle sizes on the order of micrometers, ${ }^{12}$ to continuous thin films. ${ }^{13}$ Mesostructured sol-gel thin films formed by the rapid dipcoating method have been made with hexagonal, lamellar, and cubic structures that possess a high degree of long- range order. The starting point of the process is a solution containing all of the components, molecular precursor, solvent(s), and surfactant, that assemble to form the final solid (Figure 3). All of the steps leading to the final material, including micelle formation and the hydrolysis and condensation reactions that form the silica, occur above the sol as the substrate is pulled from the liquid. As the substrate is withdrawn at a constant rate from the solution, the processes occur at different heights and times; the spatial separation enables the various stages of the process to be interrogated.

The application of spectroscopic probe methods has been extremely useful for understanding the synthesis of mesosotructured films because the information obtained includes details of micelle formation, chemical changes occurring in the film, and film drying. The initial studies used cetyltrimethylammonium bromide (CTAB) as the structure-directing agent for silica films and 2- $p$-toluidinyl naphthalene-6-sulfonate (TNS) to determine the point during the film deposition process when sufficient evaporation had occurred, so that the critical micelle concentration (cmc) was reached. ${ }^{13}$ In other work (Figure 3), we combined two spectroscopic probe molecules, pyranine and pyrene, in the study of mesostructured films templated with sodium dodecyl sulfate (SDS) ${ }^{14}$ Luminescence peaks from pyranine showed that the preferential loss of alcohol gives a water-rich film near the end of the film deposition process. The second probe molecule, pyrene, is sensitive to the polarity of its immediate environment and has been used to monitor micelle formation. The relative intensities of the vibronic emission bands (Figure 3 ) undergo systematic variations as the local surroundings change from the polar sol to the nonpolar micelle. These micelles formed within a few seconds after the substrate left the sol. 


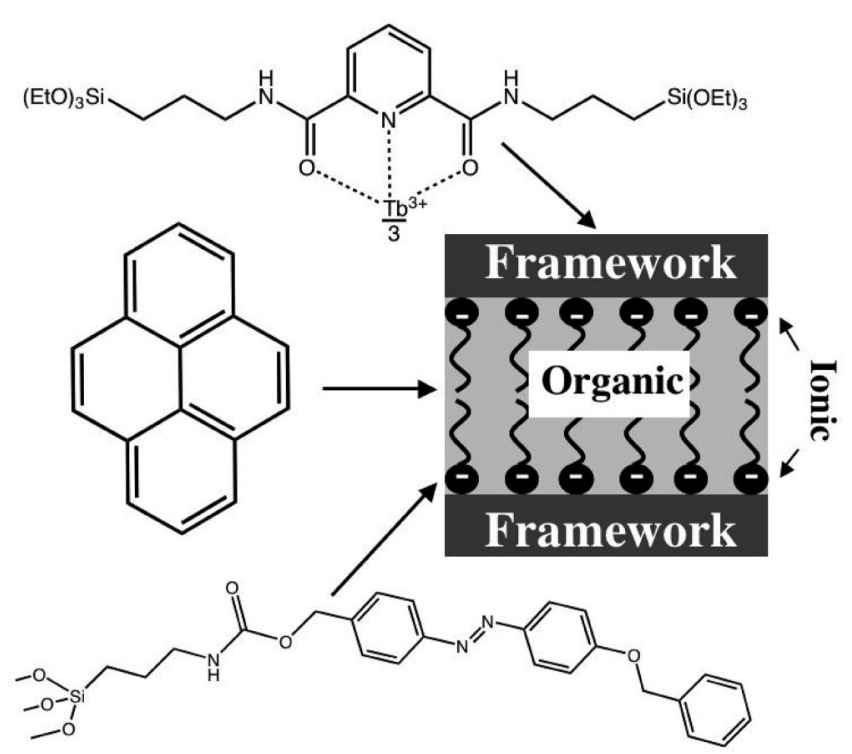

FIGURE 4. Examples of molecules placed in specific regions of a mesostructured film. The different regions are the silica framework, the organic interior of the micelle, and the ionic interface between them. The strategies for placing specific molecules in the different regions are discussed in the text.

Rigidochromic probe molecules were also used successfully to study the properties of mesostructured silica films. In this work, the probe molecule, $\mathrm{ClRe}(\mathrm{CO})_{3}-2,2^{\prime}-$ bipyridine, served dual functions: its emission band maximum shifts to a higher energy with an increasing rigidity of its environment, and simultaneously, the fluorescence depolarization measures the mobility of the probe molecule itself. ${ }^{15}$ In freshly prepared mesostructured films, the probe molecules reside in both the silica framework (in a rigid environment) and the ionic interface region near the surfactant head groups (in a nonrigid environment) as shown by the rigidochromic shift. The fluorescence depolarization confirms the rigidity; the molecule is fixed in position in the silica framework but rotates in the interface region. The spectra of the probe in dried, and frozen films show that maximum rigidity is achieved at low temperatures.

\section{Deliberate Placement of Probe Molecules}

The use of nonpolar spectroscopic probe molecules, pyrene and TNS, in the above studies suggested the possibility of deliberately placing molecules within specific regions of mesostructured films. Our work over the past few years has demonstrated that, by using appropriate strategies, it is possible to place a wide variety of dopant molecules in specific regions of mesostructured materials. $^{16,17}$ At the end of this section, we describe an application of the deliberate placement strategy, where energy transfer occurs between separated donor and acceptor molecules. ${ }^{18}$

Mesostructured materials consist of three distinct regions. The regions are shown in Figure 4. The silica network that holds the material together is termed the framework. The thickness of the framework can be controlled by both the sol-gel chemistry and the speed of the dip-coating process. The region containing the hydrophobic long-chain hydrocarbon tails of the surfactant is called the organic region. It is a planar sheet in the lamellar materials and a cylinder in the hexagonal materials. The dimensions of the organic region are controlled by the length of the hydrocarbon tail of the surfactant used in the preparation. The third region that contains the ionic head group of the surfactant and the counterions is called the ionic region. It is a few angstroms thick and forms the interface between the framework and the organic region. In the as-deposited films, it also contains residual water as well as ions from the acid catalyst and buffer if they were used in the preparation.

Strategies for Placing Active Molecules in Desired Regions. We have used three synthetic strategies for placing the donor and acceptor in the structured film. ${ }^{16,17}$ These strategies are (a) physically immobilizing the desired molecule in the desired region ("philicity" strategy), (b) chemically forming the framework composed of the molecule (bonding strategy), and (c) chemically bonding one part of the molecule to the framework/ionic interface and incorporating the other parts elsewhere ("bifunctional" strategy). Examples of these strategies are shown in Figure 4.

Physical immobilization of molecules utilizes the site "philicity" (or local solubility) of the molecule. Hydrophobic molecules will reside in the organic region of the final material; ionic molecules will reside in the ionic region; and neutral polar molecules will reside in the framework. The best examples of the use of this simple strategy involve hydrophobic molecules, where pyrene was used as a probe of micelle formation ${ }^{14}$ and rhodamine 6G (R6G) was used as an energy acceptor as discussed below.

The method of choice for deliberately placing the donor or acceptor in the framework region of the material is to make the active molecule the building block of the framework. In this method, the active molecule itself will form the framework. ${ }^{16,17}$ The major requirements are that the molecule must itself undergo hydrolysis and condensation to form the framework and it also must allow for templating by the micelles to occur before the final framework is formed. One successful approach has involved rare earth complexes of Eu and $\mathrm{Tb}$, chelated by an alkoxysilylated ligand that becomes incorporated in the silicate framework (Figure 4).

The ideal method for placing an active molecule at the interface between the framework and the ionic region is to chemically bond it to the outside of the framework. This method can be achieved by making one end of the molecule a trialkoxysilane and the other end hydrophobic. ${ }^{16,17}$ This approach has been successfully applied to ruthenium complexes.

Application of Deliberate Placement: Energy Transfer. A recent example of functionality induced in nanostructured films is the energy transfer between spatially separated donor and acceptor molecules. ${ }^{18}$ Quantitative measurements of energy transfer, in conjunction with spec- 
troscopic wavelength shifts, demonstrated that spatial separation on the nanoscale actually does occur. In addition, energy transfer was used as a molecular ruler to study material properties.

The silicate thin films that were used in the energytransfer studies had a 2D hexagonal structure templated by CTAB. The films were doped simultaneously with two different luminescent molecules, R6G and a Tb complex. ${ }^{18}$ Luminescence spectroscopy showed that R6G, the molecule chosen as the energy acceptor, was incorporated in the surfactant micelles and that the Tb complex chelated by an alkoxysilylated ligand was incorporated in the silicate framework. Steady-state luminescence spectra, lifetime measurements of R6G luminescence, and lifetime measurements of the emission of the Tb complex provided evidence of energy transfer. The Tb luminescence lifetime measurements were used to calculate the distance between $\mathrm{Tb}$ and R6G in nanostructured films according to the Förster model. At the lowest R6G/Tb concentration ratio studied, 1.6:100 $\mathrm{R} 6 \mathrm{G} / \mathrm{Tb}$, a large average $\mathrm{Tb}-\mathrm{R} 6 \mathrm{G}$ distance of $R=65 \AA$ was calculated. This distance decreased as the R6G concentration increased until it reached a minimum value of $29 \AA$ at 20:100 R6G/Tb.

\section{Functional Properties of Dye-Doped Sol-Gel Materials}

In parallel with research on spectroscopic probes was a significant effort at creating a new generation of optical materials based on doping-specific organic and organometallic molecules in sol-gel-derived matrices. ${ }^{19}$ The sol-gel process is sufficiently benign that the molecules dissolved in the inorganic glass matrix effectively retain their characteristic solution properties. Using the sol-gel doping approach, a wide variety of materials with designed optical properties were produced. Some of the more significant properties demonstrated by dye-doped sol-gel materials are photochromism, ${ }^{6}$ laser action, ${ }^{20}$ surface-enhanced Raman emission, ${ }^{21}$ and a variety of nonlinear optical properties. ${ }^{22}$ As recent review papers indicate ${ }^{22,23}$ this field continues to generate considerable interest, with particular emphasis on the technological development of optical communication devices that use sol-gel-derived inorganic/organic hybrid materials.

The use of sol-gel materials for sensor applications has emerged over the past decade because of the ability to adapt this approach as a optochemical transducer platform for a wide range of fields ranging from food analysis and environmental monitoring to drug detection and biosensing. As described in the following section, some of these areas are based on the encapsulation of biomolecules rather than probe molecules. However, the recognition that sol-gel materials could serve as sensors stems from the spectroscopic probe studies involving pyranine. ${ }^{7}$ In this work, pyranine was effectively trapped inside the solid monolith, but it still responded to the external solvent composition. The enabling microstructural feature, which was not appreciated until this study, was that the pore network was interconnected from the interior to the exterior. This sensitivity to external solvents and their dissolved species led to the demonstration of optical sensors for $\mathrm{pH}$ and various chemicals. ${ }^{6,24}$ The ability to couple sol-gel materials processing with ink jet printing has been an important step toward commercialization.

\section{Encapsulation of Biological Molecules in Sol-Gel Matrices}

The emergence of sol-gel methods for immobilizing biomolecules has two points of origin. First, it was abundantly clear from the research on encapsulating organic and organometallic molecules that the ability to dope sol-gel glasses was not limited to just a few systems. As long as the dopant was soluble in the aqueous or alcohol-based solvent, it could be incorporated within the sol-gel matrix and, in most cases, effectively impart its properties to the resulting solid. It would seem that an analogous approach would be feasible for biomolecular dopants. Second, there was a growing interest in using sol-gel materials for optically based chemical sensors. The ability to dope biomolecules in the sol-gel matrix would not only lead to better specificity and sensitivity but also enable biosensing applications. Today, the field of sol-gel immobilization of biomolecules is a very active one. ${ }^{25}$ The field has grown dramatically because there is now the ability to routinely use sol-gel methods to encapsulate biomolecules ranging in size from small proteins of a few nanometers to whole cells of several micrometers. The field has diversified from soluble proteins to membranebound proteins and from sensor applications to new directions, such as high throughput drug screening and energy storage. While the encapsulation of cells was one of the first studies in the field, ${ }^{26}$ after only modest interest for a number of years, ${ }^{27}$ the topic of cell encapsulation is rapidly gaining renewed interest in part because of new synthetic routes. ${ }^{28,29}$ In this section, we highlight some of the features that have led to the emergence of sol-gel encapsulation of biomolecules. We conclude the section with a discussion of future directions.

The earliest examples of biomolecule encapsulation in sol-gel matrices were largely directed at the encapsulation of soluble proteins. ${ }^{30,31}$ The fundamental question addressed in these studies was whether proteins retained their characteristic reactivity and function when encapsulated in the sol-gel-derived $\mathrm{SiO}_{2}$ matrix. These first biomolecule encapsulation studies showed that, as long as one used synthesis conditions that avoided protein denaturation, the immobilized enzymes retained their catalytic ability, proteins exhibited their metal-exchange properties, heme proteins displayed redox behavior and ligand binding, and antibodies retained their binding affinity. ${ }^{6,32}$

The ability to prepare optically transparent materials was an important consideration in many studies because it was possible to monitor the spectroscopic response of the sol-gel material throughout the processes of gelation, aging, and drying. The visible electronic spectra of metalloproteins were especially useful for these studies be- 


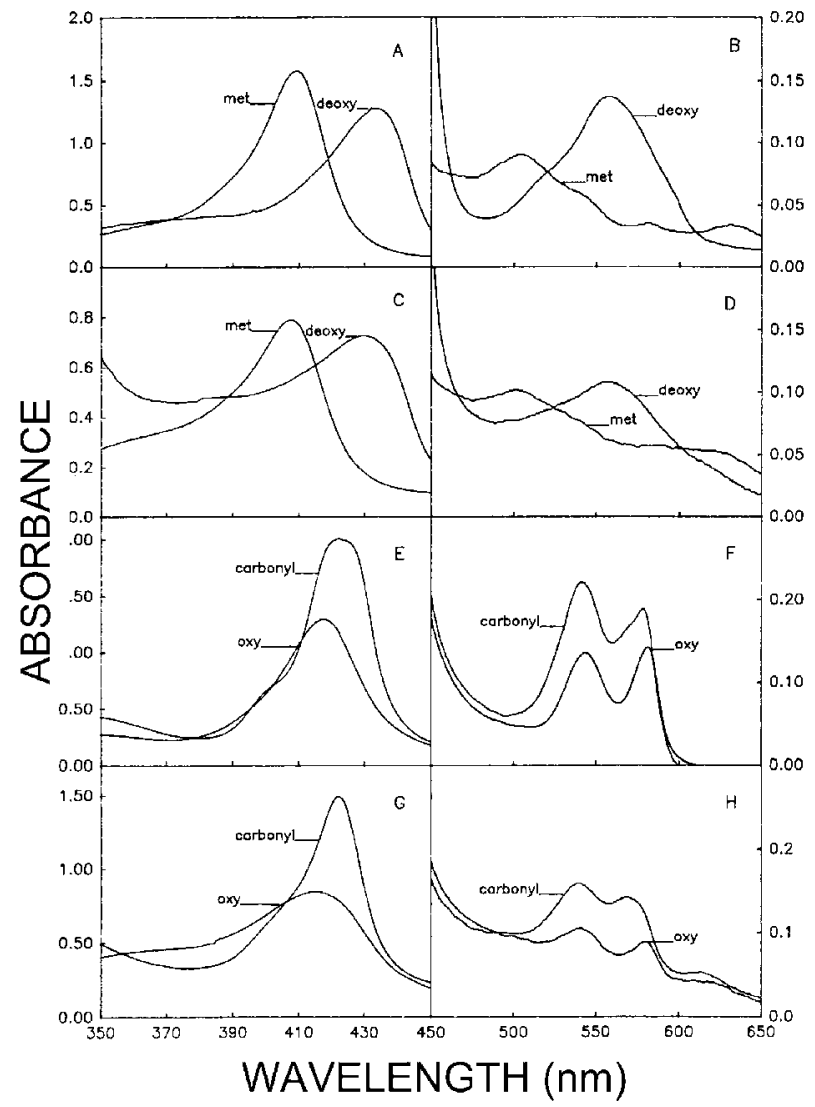

FIGURE 5. Absorption spectra for met-Mb, deoxy-Mb, carbonyl$M b$, and oxy-Mb in solution (A, B, E, and $F)$ and in the sol-gel matrix $(C, D, G$, and $H) .{ }^{31}$ The spectral similarity between the encapsulated and dissolved proteins was an early demonstration that sol-gel immobilization did not destroy the protein structure.

cause the spectra are sensitive to protein conformation and metal-ion occupancy. ${ }^{33}$ The binding of various ligands to myoglobin $(\mathrm{Mb})$ is shown in Figure 5. The absorption spectra for sol-gel-encapsulated deoxy-Mb, carbonyl-Mb, and oxy-Mb exhibit the same spectroscopic features as the corresponding solutions. The similarity in spectra between encapsulated proteins and those in solution demonstrated very early that it was possible to encapsulate proteins in sol-gel matrices without altering the protein structure. It should be noted, however, that the sol-gel encapsulation process does not always trap the protein in its native conformation, because there are instances where intermediate conformations are trapped. ${ }^{34}$ In addition to enabling studies of proteins in metastable conformations, sol-gel encapsulation also enables researchers to study the effect of confinement on the protein structure and stability. ${ }^{35}$

An important contribution in these early studies was the development of relatively simple synthetic procedures that successfully avoid protein denaturation. A two-step approach was devised in which TMOS was prehydrolyzed, generally without the addition of alcohol, followed by the addition of buffer to bring the $\mathrm{pH}$ to a range where the protein was stable. ${ }^{31,36}$ A benefit of this approach was the ability to produce transparent monoliths, usually formed in spectroscopy cuvettes, to facilitate in situ monitoring of the spectroscopic prop-

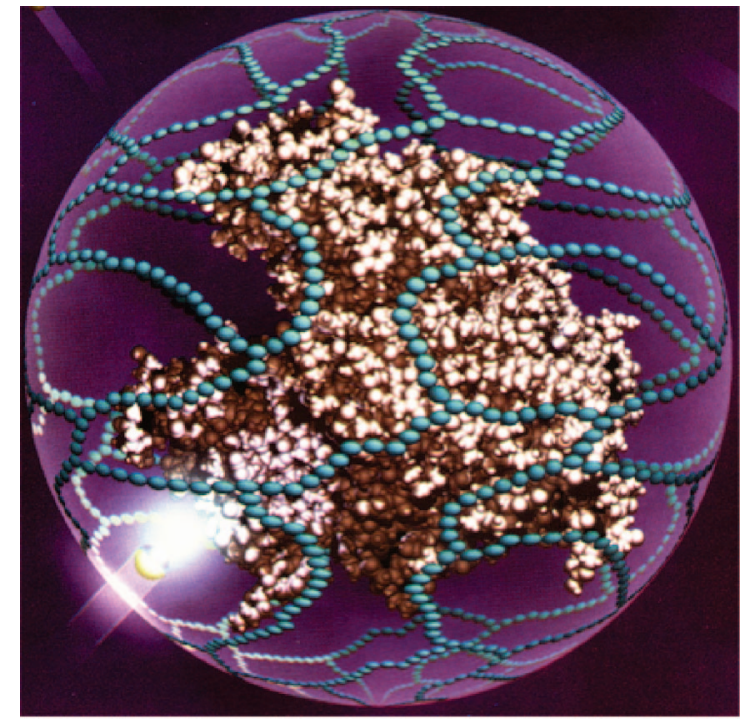

FIGURE 6. Idealized microstructure of a sol-gel glass containing a biomolecule. The porous sol-gel glass is tailored so that large protein macromolecules are immobilized in the matrix, while small molecules (i.e., the analyte to be detected) are free to enter and diffuse through the porous network. ${ }^{32}$

erties of the protein. As the biomolecule immobilization field has continued to grow, there is interest in developing matrices for biosystems (e.g., phospholipids and whole cells) that cannot tolerate alcohols. As a result, a number of approaches have emerged in recent years that greatly lower the alcohol content and generally provide improvements in microstructure control. These include aqueous synthesis routes based on sodium silicate $^{37}$ and the use of biocompatible silane precursors containing bound sugar moieties. ${ }^{38}$

Biosensor Applications and Enhanced Stability. The early studies with soluble proteins established two key features. First, materials based on the sol-gel encapsulation of biomolecules represented a new generation of materials for highly selective and sensitive biosensors. The second key attribute is the enhanced stability of the protein that arises from the encapsulation process. Trapping the protein in the silica cage effectively suppresses protein unfolding and avoids denaturation. Figure 6 illustrates how the biomolecule is confined within a large pore and yet is able to respond to small analyte molecules because of the inherent porosity of the sol-gel matrix.

Biosensors. Biosensors have proven to be an extremely successful direction for sol-gel-encapsulated biomolecules. ${ }^{39}$ Despite being trapped in the matrix, biological molecules retain the catalytic, recognition, and transduction functions that make the resulting sol-gel materials ideal for the design of sensors over a broad range of areas, including medical and health care, environmental monitoring, industrial processing, food quality, toxic chemicals, and explosives. Finally, it is important to mention that sol-gel-based biosensors have moved beyond the research stage, and commercialization efforts are well underway.

Glucose sensors based on the encapsulation of glucose oxidase $\left(\mathrm{GO}_{x}\right)$ have received more attention than any other 
encapsulated enzyme system, and these studies underscore the versatility of using sol-gel methods for biosensing. ${ }^{32,39}$ Both optical- and electrochemical-sensing schemes have been demonstrated, involving a variety of matrices based on sol-gel synthesis. Sol-gel-based biosensors have been reported for a wide variety of oxidoreductases. In the case of dehydrogenases, a coenzyme of nicotinamide adenine dinucleotide (NAD) or nicotinamide adenine dinucleotide phosphate (NADP) is required, and such systems have been shown to function effectively in sol-gel matrices. ${ }^{40}$ One advantage here for optical detection is that the reduced form of the coenzyme (e.g., $\mathrm{NADH}$ ) is luminescent, while the oxidized form (e.g., $\mathrm{NAD}^{+}$) is not. This distinguishing characteristic was used in the development of a enzyme-based biosensor for detection of the neurotransmitter, glutamate.

Another biosensor area where sol-gel methods have had a significant impact is in the development of antibodybased sensors. ${ }^{39,41}$ The attractiveness of using immunoassays stems from the highly selective recognition of target analytes as well as the ability to develop antibodies to an extraordinary range of antigens. One advantage of the sol-gel approach is that it offers a generic immobilization strategy adaptable to different antibody systems and requires one or two steps rather than the multiple steps involved in the usual sandwich-type assay. Moreover, as was the case with enzymatic sensors, sol-gel approaches are readily adapted to optical- and electrochemicaldetection methods. Recent studies have extended sol-gel immunoassays to explosive detection, ${ }^{42}$ pathogens, herbicides, and hormones. $^{25}$

Protein Stabilization. One of the most important benefits of sol-gel immobilization is the ability to stabilize biomolecules through encapsulation. There is indirect evidence that a biomolecule designs a self-specific pore as the silicate network forms around it during sol-gel hydrolysis and condensation reactions. ${ }^{33}$ This silicate "cage" defines the pore according to the size and shape requirements of the biomolecule. Consequently, the biomolecule prevents its surrounding pore from collapsing, while the matrix protects the biomolecule from unfolding and aggregation. In addition, the matrix prevents contact with proteases or microorganisms. In research to date, enhanced stability has been reported in terms of thermal, storage, and chemical stability for sol-gel-immobilized biomolecules. Among the more significant results are those reported for three flavoprotein oxidases, glucose oxidase $\left(\mathrm{GO}_{x}\right)$, lactate oxidase $\left(\mathrm{LO}_{x}\right)$, and glycolate oxidase $\left(\mathrm{GLyO}_{x}\right),{ }^{43}$ cytochrome c (cyt c) ${ }^{33}$ phosphatases, ${ }^{30} \alpha$-lactalbumin, ${ }^{35}$ and creatine kinase. ${ }^{44}$ The extent of stabilization for $\mathrm{GO}_{x}$ was particularly impressive, because the halflife at $63{ }^{\circ} \mathrm{C}$ was increased 200 -fold upon sol-gel encapsulation as compared to the enzyme in water.

The storage stability of sol-gel-encapsulated biomolecules was noted in the first studies, ${ }^{30}$ and subsequent work has quantified this behavior. A more recent example is the study reported using creatine kinase. ${ }^{44}$ In solution, the enzymatic activity decreases to $\sim 50 \%$ of its initial activity within 10 days, while the sol-gel-encapsulated material retained some $90 \%$ of its initial activity after 5 months. Recent results show that sol-gel encapsulation effectively prevents proteases from affecting the protonpumping characteristics of bacteriorhodopsin. ${ }^{45}$ Closely related to the storage stability is the chemical stability of sol-gel-encapsulated biomolecules. The isolation of proteins within pores constrains their translational mobility, thus avoiding aggregation despite exposing the sol-gelencapsulated protein to solvent conditions that commonly promote protein aggregation $(\mathrm{pH}$, alcohol, and ionic strength). The matrix may also prevent the protein from unfolding, and the importance of confined water within the pores has also been suggested to be an important consideration. ${ }^{35}$

Future Directions. Recent reviews have emphasized the increased interest in the sol-gel encapsulation of whole cells. ${ }^{25,28}$ As indicated previously, the development of new synthetic approaches has overcome many of the alcohol and $\mathrm{pH}$ limitations experienced in earlier work, enabling the field to make progress in a number of areas. With the ability of cells to be used in a variety of areas, including biosynthesis, sensing, and as scaffolds for cell and tissue growth, it is evident that the cell encapsulation area will be an active one in the coming years.

Another emerging area for the bioencapsulation field is that of incorporating membrane-bound proteins. Membrane-receptor proteins play a vital role in controlling cell processes, and the ability to immobilize these proteins in inorganic matrices represents a significant step forward in developing a new generation of biologically active materials. A few studies have now appeared that highlight some future opportunities. These first reports include the successful sol-gel encapsulation of the transmembrane peptide ion-channel gramicidin A, a ligand-gated ion channel (nicotinic acetylcholine receptor), and a G-protein-coupled receptor (dopamine D2 receptor). ${ }^{46}$ These studies point to the promise of applying sol-gel methods in areas such as high-throughput drug screening and for new generations of sensors. Another recent paper, which reported the encapsulation of both bacteriorhodopsin and $F_{0} F_{1}$ ATP synthase, successfully demonstrated the use of a photo-induced proton gradient for the biosynthesis of ATP. ${ }^{45}$ This work underscores the prospect of using membrane-associated proteins to design multifunctional biocomposite materials, which enable biological modes of power generation and energy storage.

Another potential research area related to energy production is that of using sol-gel encapsulation to harness biomolecules for fuel cells. The field of biofuel cells is rapidly emerging, and the ability to fabricate electrodes with enhanced enzyme stability and operation at elevated temperatures is very attractive for biofuel cell operation. Nanostructured composite electrodes based on enzyme encapsulation and the incorporation of carbon nanotubes was recently reported. ${ }^{47}$ The enzymes, $\mathrm{GO}_{x}$ and bilirubin oxidase, maintain their biocatalytic activity in the sol-gel matrix, and the first sol-gel-based glucose-oxygen biofuel cells exhibited voltage and power density values 
comparable to that reported for the same enzyme system using traditional methods. With the ability to encapsulate whole cells, it would seem that the development of microbial fuel cells could also benefit by incorporating sol-gel encapsulation strategies.

\section{Summary}

This Account has traced the development of molecules entrapped in silica prepared by sol-gel methods, from the first reports of the incorporation of organic molecules to the design and synthesis of advanced functional materials. The new generation of materials, which involves such areas as nanostructures, ${ }^{13}$ self-assembly, ${ }^{18}$ molecular machines, ${ }^{48}$ and biosystems, ${ }^{25}$ attests to the vitality of this field.

We are grateful for the support of our research by the National Science Foundation (DMR-0103952).

\section{References}

(1) Dickey, F. H. The Preparation of Specific Adsorbents. Proc. Natl. Acad. Sci. U.S.A. 1949, 35, 227-229.

(2) Avnir, D.; Levy, D.; Reisfeld, R. The Nature of the Silica Cage as Reflected by Spectral Changes and Enhanced Photostability of Trapped Rhodamine 6G. J. Phys. Chem. 1984, 88, 5956-5959.

(3) Brinker, C. J.; Scherer, G. W. Sol-Gel Science: The Physics and Chemistry of Sol-Gel Processing; Academic Press, Inc.: San Diego, CA, 1990.

(4) Hench, L. L.; West, J. K. The Sol-Gel Process. Chem. Rev. 1990, 90, 33-72.

(5) Dunn, B.; Zink, J. I. Probes of Pore Environment and MoleculeMatrix Interactions in Sol-Gel Materials. Chem. Mater. 1997, 9, 2280-2291.

(6) Avnir, D. Organic Chemistry within Ceramic Matrices: Doped SolGel Materials. Acc. Chem. Res. 1995, 28, 328-334.

(7) Pouxviel, J. C.; Dunn, B.; Zink, J. I. Fluorescence Study of Aluminosilicate Sols and Gels Doped with Hydroxy Trisulfonated Pyrene. J. Phys. Chem. 1989, 93, 2134-2139.

(8) Winter, R.; Hua, D. W.; Song, X.; Mantulin, W.; Jonas, J. Structural and Dynamic Properties of the Sol-Gel Transition. J. Phys. Chem. 1990, 94, 2706-2713.

(9) Narang, U.; Wang, R.; Prasad, P. N.; Bright, F. V. Effects of Aging on the Dynamics of Rhodamine-6G in Tetramethyl OrthosilicateDerived Sol-Gels. J. Phys. Chem. 1994, 98, 17-22.

(10) McKiernan, J.; Pouxviel, J. C.; Dunn, B.; Zink, J. I. Rigidochromism as a Probe of Gelation and Densification of Silicon and Mixed Aluminum Silicon Alkoxides. J. Phys. Chem. 1989, 93, 2129-2133.

(11) Nishida, F.; McKiernan, J. M.; Dunn, B.; Zink, J. I. In Situ Fluorescence Probing of the Chemical Changes during Sol-Gel Thin Film Formation. J. Am. Ceram. Soc. 1995, 78, 1640-1648.

(12) Kresge, C. T.; Leonowicz, M. E.; Roth, W. J.; Vartuli, J. C.; Beck, J. S. Ordered Mesoporous Molecular-Sieves Synthesized by a Liquid-Crystal Template Mechanism. Nature 1992, 359, 710-712.

(13) Lu, Y. F.; Ganguli, R.; Drewien, C. A.; Anderson, M. T.; Brinker, C. J.; Gong, W. L.; Guo, Y. X.; Soyez, H.; Dunn, B.; Huang, M. H.; Zink, J. I. Continuous Formation of Supported Cubic and Hexagonal Mesoporous Films by Sol Gel Dip-Coating. Nature 1997, 389, 364368.

(14) Huang, M. H.; Dunn, B. S.; Zink, J. I. In Situ Luminescence Probing of the Chemical and Structural Changes During Formation of DipCoated Lamellar Phase Sodium Dodecyl Sulfate Sol-Gel Thin Films. J. Am. Chem. Soc. 2000, 122, 3739-3745.

(15) Franville, A. C.; Dunn, B.; Zink, J. I. Molecular Motion and Environmental Rigidity in the Framework and lonic Interface Regions of Mesostructured Silica Thin Films. J. Phys. Chem. B 2001, 105, 10335-10339.

(16) Hernandez, R.; Franville, A. C.; Minoofar, P.; Dunn, B.; Zink, J. I. Controlled Placement of Luminescent Molecules and Polymers in Mesostructured Sol-Gel Thin Films. J. Am. Chem. Soc. 2001, 123, $1248-1249$.

(17) Minoofar, P. N.; Hernandez, R.; Chia, S.; Dunn, B.; Zink, J. I.; Franville, A. C. Placement and Characterization of Pairs of Luminescent Molecules in Spatially Separated Regions of Nanostructured Thin Films. J. Am. Chem. Soc. 2002, 124, 14388-14396.
(18) Minoofar, P. N.; Dunn, B. S.; Zink, J. I. Multiply Doped Nanostructured Silicate Sol-Gel Thin Films: Spatial Segregation of Dopants, Energy Transfer, and Distance Measurements. J. Am. Chem. Soc. 2005, 127, 2656-2665.

(19) Dunn, B.; Zink, J. I. Optical-Properties of Sol-Gel Glasses Doped with Organic-Molecules. J. Mater. Chem. 1991, 1, 903-913.

(20) McKiernan, J. M.; Yamanaka, S. A.; Dunn, B.; Zink, J. I. Spectroscopy and Laser Action of Rhodamine-6G Doped Aluminosilicate Xerogels. J. Phys. Chem. 1990, 94, 5652-5654.

(21) Akbarian, F.; Dunn, B. S.; Zink, J. I. Surface-Enhanced RamanSpectroscopy Using Photodeposited Gold Particles in Porous SolGel Silicates. J. Phys. Chem. 1995, 99, 3892-3894.

(22) Innocenzi, P.; Lebeau, B. Organic-Inorganic Hybrid Materials for Non-linear Optics. J. Mater. Chem. 2005, 15, 3821-3831.

(23) Reisfeld, R.; Weiss, A.; Saraidarov, T.; Yariv, E.; Ishchenko, A. A. Solid-State Lasers Based on Inorganic-Organic Hybrid Materials Obtained by Combined Sol-Gel Polymer Technology. Polym. Adv. Technol. 2004, 15, 291-301.

(24) MacCraith, B. D.; McDonagh, C. Enhanced Fluorescence Sensing Using Sol-Gel Materials. J. Fluoresc. 2002, 12, 333-342.

(25) Avnir, D.; Coradin, T.; Lev, O.; Livage, J. Recent Bio-applications of Sol-Gel Materials. J. Mater. Chem. 2006, 16, 1013-1030.

(26) Carturan, G.; Campostrini, R.; Dire, S.; Scardi, V.; De Altgeriis, E. Inorganic Gels for Immobilization of Biocatalysts-Inclusion of Invertase-Active Whole Cells of Yeast (Saccharomyces cerevisiae) into Thin Layers of $\mathrm{SiO}_{2}$ Gel Deposited on Glass Sheets. J. Mol. Catal. 1989, 57, L13-L16.

(27) Chia, S. Y.; Urano, J.; Tamanoi, F.; Dunn, B.; Zink, J. I. Patterned Hexagonal Arrays of Living Cells in Sol-Gel Silica Films. J. Am. Chem. Soc. 2000, 122, 6488-6489.

(28) Carturan, G.; Dal Toso, R.; Boninsegna, S.; Dal Monte, R. Encapsulation of Functional Cells by Sol-Gel Silica: Actual Progress and Perspectives for Cell Therapy. J. Mater. Chem. 2004, 14, 2087-2098.

(29) Baca, H. K.; Ashley, C.; Carnes, E.; Lopez, D.; Flemming, J.; Dunphy, D.; Singh, S.; Chen, Z.; Liu, N. G.; Fan, H. Y.; Lopez, G. P.; Brozik, S. M.; Werner-Washburne, M.; Brinker, C. J. Cell-Directed Assembly of Lipid-Silica Nanostructures Providing Extended Cell Viability. Science 2006, 313, 337-341.

(30) Braun, S.; Rappoport, S.; Zusman, R.; Avnir, D.; Ottolenghi, M. Biochemically Active Sol-Gel Glasses: The Trapping of Enzymes. Mater. Lett. 1990, 10, 1-5.

(31) Ellerby, L. M.; Nishida, C. R.; Nishida, F.; Yamanaka, S. A.; Dunn, B.; Valentine, J. S.; Zink, J. I. Encapsulation of Proteins in Transparent Porous Silicate-Glasses Prepared by the Sol-Gel Method. Science 1992, 255, 1113-1115.

(32) Dave, B. C.; Dunn, B.; Valentine, J. S.; Zink, J. I. Sol-Gel Encapsulation Methods for Biosensors. Anal. Chem. 1994, 66, A1120-A1127.

(33) Lan, E. H.; Dave, B. C.; Fukuto, J. M.; Dunn, B.; Zink, J. I.; Valentine, J. S. Synthesis of Sol-Gel Encapsulated Heme Proteins with Chemical Sensing Properties. J. Mater. Chem. 1999, 9, 45-53.

(34) Khan, I.; Shannon, C. F.; Dantsker, D.; Friedman, A.; Perez-Gonzalezde-Apodaca, J.; Friedman, J. M. Sol-Gel Trapping of Functional Intermediates of Hemoglobin: Geminate and Bimolecular Recombination Studies. Biochemistry 2000, 39, 16099-16109.

(35) Eggers, D. K.; Valentine, J. S. Molecular Confinement Influences Protein Structure and Enhances Thermal Protein Stability. Protein Sci. 2001, 10, 250-261.

(36) Dunn, B.; Miller, J. M.; Dave, B. C.; Valentine, J. S.; Zink, J. I. Strategies for Encapsulating Biomolecules in Sol-Gel Matrices. Acta Mater. 1998, 46, 737-741.

(37) Bhatia, R. B.; Brinker, C. J.; Gupta, A. K; Singh, A. K. Aqueous SolGel Process for Protein Encapsulation. Chem. Mater. 2000, 12, 2434-2441.

(38) Sui, X. H.; Cruz-Aguado, J. A.; Chen, Y.; Zhang, Z.; Brook, M. A.; Brennan, J. D. Properties of Human Serum Albumin Entrapped in Sol-Gel Derived Silica Bearing Covalently Tethered Sugars. Chem. Mater. 2005, 17, 1174-1182.

(39) Gill, I. Bio-doped Nanocomposite Polymers: Sol-Gel Bioencapsulates. Chem. Mater. 2001, 13, 3404-3421.

(40) Rickus, J. L.; Dunn, B.; Zink, J. I. Optically Based Sol-Gel Biosensor Materials. In Optical Biosensors; Ligler, F. S., Rowe-Taitt, C. A., Eds.; Elsevier Science: Amsterdam, The Netherlands, 2002.

(41) Wang, R.; Narang, U.; Prasad, P. N.; Bright, F. V. Affinity of Antifluorescein Antibodies Encapsulated within a Transparent SolGel Glass. Anal. Chem. 1993, 65, 2671-2675.

(42) Lan, E. H.; Dunn, B. S.; Zink, J. I. Sol-Gel Encapsulated Antitrinitrotoluene Antibodies in Immunoassays for TNT. Chem. Mater. 2000, 12, 1874-1878.

(43) Chen, Q.; Kenausis, G. L.; Heller, A. Stability of Oxidases Immobilized in Silica Gels. J. Am. Chem. Soc. 1998, 120, 4582-4585.

(44) Nguyen, D. T.; Smit, M.; Dunn, B.; Zink, J. I. Stabilization of Creatine Kinase Encapsulated in Silicate Sol-Gel Materials and Unusual Temperature Effects on Its Activity. Chem. Mater. 2002, 14, 43004306. 
(45) Luo, T.-J. M.; Soong, R.; Lan, E.; Dunn, B.; Montemagno, C. Photoinduced Proton Gradients and ATP Biosynthesis Produced by Vesicles Encapsulated in a Silica Matrix. Nat. Mater. 2005, 4, 220224.

(46) Besanger, T. R.; Easwaramoorthy, B.; Brennan, J. D. Entrapment of Highly Active Membrane-Bound Receptors in Macroporous SolGel Derived Silica. Anal. Chem. 2004, 76, 6470-6475.
(47) Lim, J.; Malati, P.; Bonet, F.; Dunn, B. Nanostructured Sol-Gel Electrodes for Biofuel Cells. J. Electrochem. Soc. 2007, 154, A140A145.

(48) Saha, S.; Leung, K. C.-F.; Nguyen, T. D.; Stoddart, J. F.; Zink, J. I. Nanovalves. Adv. Funct. Mater. 2007, 17, 685-693.

AR700033B 